\title{
Long-term prognostic value of stress
} perfusion cardiovascular magnetic resonance in patients without known coronary artery disease

Théo Pezel ${ }^{1,2}$, Thierry Unterseeh ${ }^{1}$, Marine Kinnel ${ }^{1}$, Thomas Hovasse ${ }^{1}$, Francesca Sanguineti ${ }^{1}$, Solenn Toupin ${ }^{3}$, Stéphane Champagne ${ }^{1}$, Philippe Garot ${ }^{1}$ and Jérôme Garot ${ }^{1 *}$ (1)

\begin{abstract}
Background: To assess the incremental long-term prognostic value of vasodilator stress perfusion cardiovascular magnetic resonance (CMR) in patients without known coronary artery disease (CAD).

Methods: Between 2010 and 2011, consecutive patients with cardiovascular risk factors without known CAD referred for stress CMR were followed for the occurrence of major adverse cardiac events (MACE), defined by cardiovascular mortality or recurrent non-fatal myocardial infarction (MI). Uni- and multivariable Cox regressions were performed to determine the prognostic value of ischemia and unrecognized MI defined by sub-endocardial or transmural late gadolinium enhancement (LGE).

Results: Among 2,295 patients without known CAD, 2058 (89.7\%) (71.2 \pm 12.5 years; 37.5\% males) completed the follow-up (median [IQR]: 8.3 [7.3-8.7] years), and 203 had MACE (9.9\%). Using Kaplan-Meier analysis, ischemia and unrecognized MI were associated with MACE (hazard ratio, HR: 4.64 95\% Cl: 3.69-6.17 and HR: 2.88; 95\% Cl: 2.08-3.99, respectively; both $\mathrm{p}<0.001$ ). In multivariable stepwise Cox regression, ischemia and unrecognized $\mathrm{MI}$ were independent predictors of MACE $(\mathrm{HR}=3.71 ; 95 \% \mathrm{Cl} 2.73-5.05, p<0.001$ and $\mathrm{HR}=1.73 ; 95 \% \mathrm{Cl} 1.22-2.45, p=0.002$; respectively) and cardiovascular mortality (HR: 3.13; 95\% Cl: 2.17-4.51, $\mathrm{p}<0.001$ and $\mathrm{HR}=1.73 ; 95 \% \mathrm{Cl} 1.15-2.62, p=0.009$; respectively). The addition of ischemia and unrecognized $\mathrm{Ml}$ led to an improved model discrimination for MACE (change in $C$ statistic from 0.61 to $0.72 ; \mathrm{NRI}=0.431 ; \mathrm{IDI}=0.053)$.

Conclusions: Inducible ischemia and unrecognized MI identified by stress CMR have incremental long term prognostic value for the incidence of MACE in patients without known CAD over traditional risk factors and left ventricular ejection fraction.
\end{abstract}

Keywords: Cardiovascular magnetic resonance, Stress testing, Ischemia, Unrecognized myocardial infarction, Perfusion

\footnotetext{
${ }^{*}$ Correspondence: jgarot@angio-icps.com

${ }^{1}$ CMR Department, Institut Cardiovasculaire Paris Sud, Cardiovascular Magnetic Resonance Laboratory, Hôpital Privé Jacques CARTIER, Ramsay Santé, 6 Avenue du Noyer Lambert, 91300 Massy, France

Full list of author information is available at the end of the article
}

\section{Introduction}

The primary prevention to stratify cardiovascular risk of subjects without known cardiovascular disease (CAD) is crucial for public health and health care costs [1]. To date, decisions regarding the management of these individuals have relied mostly on the assessment of traditional risk original author(s) and the source, provide a link to the Creative Commons licence, and indicate if changes were made. The images or other third party material in this article are included in the article's Creative Commons licence, unless indicated otherwise in a credit line to the material. If material is not included in the article's Creative Commons licence and your intended use is not permitted by statutory regulation or exceeds the permitted use, you will need to obtain permission directly from the copyright holder. To view a copy of this licence, visit http://creativecommons.org/licenses/by/4.0/. The Creative Commons Public Domain Dedication waiver (http://creativeco mmons.org/publicdomain/zero/1.0/) applies to the data made available in this article, unless otherwise stated in a credit line to the data. 
factors. However, noninvasive cardiac stress testing may also play an important role for risk stratification, and therefore constitutes a cornerstone of the management of these subjects in the European and American guidelines $[2,3]$.

Stress cardiovascular magnetic resonance (CMR) imaging has emerged as a cost-effective modality for the diagnosis of CAD, and for risk stratification of cardiovascular events through the detection of both inducible myocardial ischemia and myocardial scar [4-7]. Prior studies have shown that beyond the major prognostic role of inducible ischemia, the depiction of an unrecognized myocardial infarction (MI) in individuals without known CAD is a strong predictor of cardiovascular events in the general population [8-11]. Recently, Nagel et al. demonstrated that a noninvasive diagnostic strategy based on stress CMR was noninferior to fractional flow reserve (FFR), in terms of outcomes in patients with suspected CAD [12].

Several large stress CMR prognostic studies have included subjects without known CAD $[4,6,7,11$, 13, 14]. In addition, targeted prognostic data in those patients without known CAD were described in specific subpopulations such as asymptomatic, elderly or obese patients [5, 15-17]. However, the incremental prognostic value of the presence of inducible myocardial ischemia and unrecognized MI vs. traditional risk factors has not been evaluated.

We hypothesized that inducible myocardial ischemia and unrecognized MI assessed by stress CMR could identify patients at higher risk for cardiovascular event in primary prevention. This study aimed to assess the long-term prognostic value of vasodilator stress perfusion CMR in subjects without known CAD and to evaluate the incremental risk stratification of stress CMR over traditional risk factors and left ventricular ejection fraction (LVEF).

\section{Methods \\ Study population}

Between December 2010 and December 2011, we conducted a single-centre longitudinal study with retrospective enrollment of consecutive patients without known CAD, referred for vasodilator stress perfusion CMR. Exclusion criteria were: (i) history of CAD [percutaneous coronary intervention (PCI) or coronary artery bypass graft $(\mathrm{CABG})$ or $\mathrm{MI}$, defined by a history of $\mathrm{MI}$ on the medical records or presence of significant $Q$ wave on 12-lead electrocardiogram (ECG) in a coronary territory]; (ii) contraindication to CMR (cerebral clips, metallic eye implant); (iii) contraindication to dipyridamole; (iv) known cardiomyopathy (e.g. hypertrophic, dilated, or infiltrative) and acute or chronic myocarditis; (v) known allergy to gadolinium-based contrast medium; and (vi) estimated glomerular filtration rate $<30 \mathrm{~mL} / \mathrm{min} / 1.73 \mathrm{~m}^{2}$. Clinical data were collected according to medical history and clinical examination on the day of stress CMR. All patients provided informed written consent. The study was approved by the local ethic committee of our institutions and conducted in accordance with the 1964 Declaration of Helsinki. This study followed the Strengthening the Reporting of Observational Studies in Epidemiology (STROBE) reporting guideline for cohort studies.

\section{Patients follow-up and clinical outcome}

The follow-up consisted of a clinical visit as part of usual care $(63 \%)$ or by direct contact with the subject or the referring cardiologist (37\%). A clinical questionnaire with a detailed description of clinical study outcomes was filled out by three senior cardiologists. Data collection was ended on January 2020. The primary outcome was the occurrence of at least one of the combined major adverse cardiac events (MACE) defined as cardiovascular mortality or non-fatal MI. The secondary outcome was cardiovascular mortality. Clinical event adjudication was based on the follow-up clinical visit or contact, with a consensus reached by two senior cardiologists. Non-fatal MI was defined by typical angina of $\geq 20 \mathrm{~min}$ duration, ECG changes, and a rise in troponin or creatine kinase level above the 99 percentile of the upper reference limit [18]. Cardiovascular mortality was defined as sudden cardiac death with documented fatal arrhythmias or any death immediately preceded by acute MI, acute or exacerbation of heart failure, or stroke. All clinical events were defined according to the published standardized definitions [19]. In patients with multiple events, only the first event was considered for event-free survival analysis. According to guidelines, an hospitalization for heart failure was defined by symptoms and/or signs of heart failure with evidence of diastolic or systolic dysfunction by echocardiography and elevated levels of brain natriuretic peptide $((\mathrm{BNP})>35 \mathrm{pg} / \mathrm{ml}$ and/or NTproBNP $>125 \mathrm{pg} / \mathrm{ml}$ )) [20]. Ventricular tachycardia was defined as documented sustained ventricular tachycardia on 12-leads ECG. Elective late coronary revascularization was defined by a revascularization occurring $>90$ days after $\mathrm{CMR}$. For patients who underwent $\mathrm{PCI}<90$ days after the index examination, peri-procedural events (MI or cardiovascular mortality) [21] were not included in the analysis.

\section{CMR protocol}

The detailed CMR protocol has already been published in our previous studies $[15,16]$. CMR was performed on a $1.5 \mathrm{~T}$ CMR scanner (MAGNETOM Espree, Siemens Healthineers, Erlangen, Germany) with an 18-channel 
anterior body coil. Long-axis (2-, 3-, and 4-chamber) and short-axis cine images encompassing the left ventricle (LV) from base to apex were obtained with a segmented retrospectively gated balanced steady state free precession (bSSFP) sequence. Vasodilatation was induced with dipyridamole injected at $0.84 \mathrm{mg} / \mathrm{kg}$ over $3 \mathrm{~min}$ for all patients. At the end of dipyridamole infusion, a bolus of gadolinium-based contrast agent $(0.1 \mathrm{mmol} /$ $\mathrm{kg}$, Dotarem ${ }^{\circledR}$, Guerbet, France) was injected at a rate of $5.0 \mathrm{ml} / \mathrm{s}$ with an injector (Optistar ${ }^{\circledR}$ Elite, Mallinckrodt). Stress perfusion imaging was performed using an ECGtriggered saturation-prepared bSSFP sequence with the following typical parameters: repetition time/echo time $(\mathrm{TR} / \mathrm{TE})=287 / 1.2 \mathrm{~ms}$, acceleration factor $=2$, field of view $=370 \times 314 \mathrm{~mm}$, matrix $=224 \times 180$, reconstructed pixel size $=1.7 \times 1.7 \times 8 \mathrm{~mm}$. A series of six slices (four short-axis views: a 2 -chamber and a 4 -chamber view) were acquired every other heartbeat. Then, theophylline was injected intravenously ( $250 \mathrm{mg}$ over $5 \mathrm{~min}$ ) to null the effect of dipyridamole. Ten minutes after contrast injection, breath-hold contrast-enhanced 3D T1-weighted inversion-recovery gradient-echo sequence was acquired with the same prescriptions to detect late gadolinium enhancement (LGE). The inversion time was individually adjusted to null normal myocardium. In case of artifacts on LGE, additional 2D single-shot bSSFP images with phase sensitive inversion recovery reconstruction were acquired. Patients were asked to refrain from caffeine at least $12 \mathrm{~h}$ before CMR. Safety was studied with clinical monitoring $1 \mathrm{~h}$ after CMR to assess major adverse events. A 12-lead ECG was performed both before and after CMR examination.

\section{CMR image analysis}

The syngo.via software (Siemens Healthineers) was used for image display and processing, and Hemolia (Clinigrid Inc., Paris, France) was used for reporting. LV volumes and function were quantified on the short-axis cine stack. Stress perfusion and LGE images were evaluated according to the 17-segment model of the American Heart Association [22]. The analysis of perfusion images was done visually by two experienced cardiologists (JG and FS) blinded to clinical and follow-up data. Inducible ischemia was defined as a subendocardial perfusion defect that (1) occurred in at least one myocardial segment, (2) persisted for at least three phases beyond peak contrast enhancement, (3) followed a coronary distribution, and (4) occurred in the absence of LGE in the same segment [13, 23-25]. An unrecognized MI was defined by LGE with ischemic patterns defined by subendocardial or transmural LGE [26]. The total number of ischemic segments was calculated for each patient. LGE was semi-quantitatively assessed using the number of LGE segments. Mild, moderate, and severe ischemia were defined as the involvement of $1-2,3-5$, and $\geq 6$ myocardial segments, respectively.

\section{Statistical analysis}

Continuous variables were expressed as mean \pm standard deviation (SD) and categorical variables as frequency with percentage. Follow-up was presented as median and interquartile range (IQR). Differences between patients with and without inducible ischemia in terms of baseline clinical and CMR characteristics were compared using the Student's t-test or the Wilcoxon rank-sum test for continuous variables and the chi-square or Fisher's exact test for categorical variables, as appropriate. Normal distribution was assessed using the Shapiro-Wilk test. Cumulative incidence rates of individual and composite outcomes were estimated using the Kaplan-Meier method and compared with the log-rank test. The proportional hazard assumption was visually assessed using Schoenfeld residuals (Additional file 1: Figure S1). Data on patients who were lost to follow-up were censored at the time of the last contact. Cox proportional hazards methods were used to identify the predictors of MACE among patients with and without ischemia. The assumption of proportional hazards ratio (HR) was verified.

The different multivariable models used for adjustment were as follows:

Model 1: used a stepwise forward Cox regression strategy to select the strongest parsimonious set of clinical covariates for MACE and cardiovascular mortality, considering all clinical covariates with a $\mathrm{p}$-value $\leq 0.2$ on univariable screening (without the presence of ischemia and unrecognized MI).

Model 2: model 1+ presence of unrecognized MI.

Model 3: model 1+ presence of unrecognized MI and presence of ischemia.

The discriminative capacity of each model for predicting MACE was determined according to the Harrell's $\mathrm{C}$-statistic at baseline and after addition of CMR-assessed ischemia and MI. The additional predictive value of the presence of ischemia and MI was calculated by the Harrell's C-statistic increment, the categorical net reclassification improvement (NRI), and the integrative discrimination index (IDI) [27]. NRI and IDI were computed at the end of follow-up using the R package "survIDINRI" [28].

In addition, the prognostic value of stress CMR in different subsamples of clinical interest was investigated by a Forest Plot. A two-tailed $\mathrm{p}$-value $<0.05$ was considered statistically significant. Statistical analysis was performed using R software, version 3.3.1 (R Project for Statistical Computing). 


\section{Results}

\section{Patients characteristics}

During the inclusion period, 2295 patients without known CAD were referred for dipyridamole stress CMR (Fig. 1). Amongst them, 2,058 patients completed the clinical follow-up and constituted our study cohort. Baseline subject characteristics and baseline CMR data are shown in Table 1. Among those 2,058 subjects (71.2 \pm 12.5 years; $37.5 \%$ males), $66.0 \%$ had hypertension, $48.9 \%$ dyslipidemia, $35.3 \%$ obesity, $34.1 \%$ diabetes mellitus, $24.9 \%$ family history of CAD, and $21.2 \%$ were smokers. Most subjects were in sinus rhythm (99.6\%). The overall study cohort had an LVEF of $52.6 \pm 12.7 \%$. An unrecognized MI was diagnosed by LGE with an ischemic pattern in $210(10.2 \%)$ patients, and inducible ischemia was detected in 267 (13.0\%) patients (Fig. 2).

Patients with ischemia were older $(74.7 \pm 11.4$ vs. $70.8 \pm 12.6$ years, $\mathrm{p}<0.001)$ and more commonly male $(59.6 \%$ vs. $34.2 \%, \mathrm{p}<0.001)$. Patients with ischemia presented a higher cardiovascular risk using the ten-year risk for fatal CAD score [29] (3.4 [1.7-6.4]\% vs. 2.4 [0.9$5.4] \%, \mathrm{p}<0.001)$ and the Framingham Risk Score $>20 \%$ risk of CAD at 10 years [30] (56.9\% vs. $31.9 \%, \mathrm{p}<0.001)$.

Of the 267 patients with ischemia, 196 (73.4\%) underwent coronary angiography with early revascularization $<90$ days after CMR. Among those, 9 patients were censored due to the recurrence of MI or cardiovascular mortality within 90 days after CMR.

\section{CMR study}

Of 2295 patients without known CAD, 2247 (97.9\%) completed the stress CMR protocol. Reasons for failure to complete CMR are presented in Fig. 1. No patient died during or shortly after CMR. There was one case of unstable angina and one patient with persistent atrial fibrillation, but no cases of transient ischemic attack, disabling stroke, ST elevation MI or sustained ventricular tachycardia after stress CMR. The main adverse events during or immediately after stress CMR were: headaches $(\mathrm{N}=276,12.3 \%)$, chest discomfort $(\mathrm{N}=198,8.8 \%)$, nausea or vomiting $(\mathrm{N}=195,8.7 \%)$, dizziness $(\mathrm{N}=54,2.4 \%)$ and angina with ECG evidence of ischemia $(\mathrm{N}=42$, $1.9 \%)$. For all patients, symptoms resolved quickly after an intravenous injection of theophylline and with additional sublingual nitrates and/or intravenous beta blockers in 29 patients (1.3\%).

\section{Prognostic value}

Among 2247 patients who underwent the stress CMR protocol, 2058 (91.6\%) completed the follow-up with a median (IQR) follow-up of 8.3 (7.3-8.7) years. There were 203 MACE (9.9\%), including 150 cardiovascular mortality (7.3\%) and 53 non-fatal MI (2.6\%). Furthermore, 296 all-cause mortality (14.4\%), 116 hospitalizations for heart failure (5.6\%), 105 elective late coronary revascularizations (5.1\%) (5 CABG), and 41 sustained documented ventricular tachycardia $(2.0 \%)$ were recorded. The annualized event rates for MACE and cardiovascular mortality, depending on the presence and severity of ischemia, are shown in Additional file 1: Figure S2.

The univariable analysis of baseline patients and CMR characteristics for the prediction of MACE and cardiovascular mortality is shown in Table 2. Age, male gender, the presence of ischemia, the number of ischemic segments, the presence of unrecognized MI, LVEF and both LV end-diastolic and end-systolic volumes indexed were

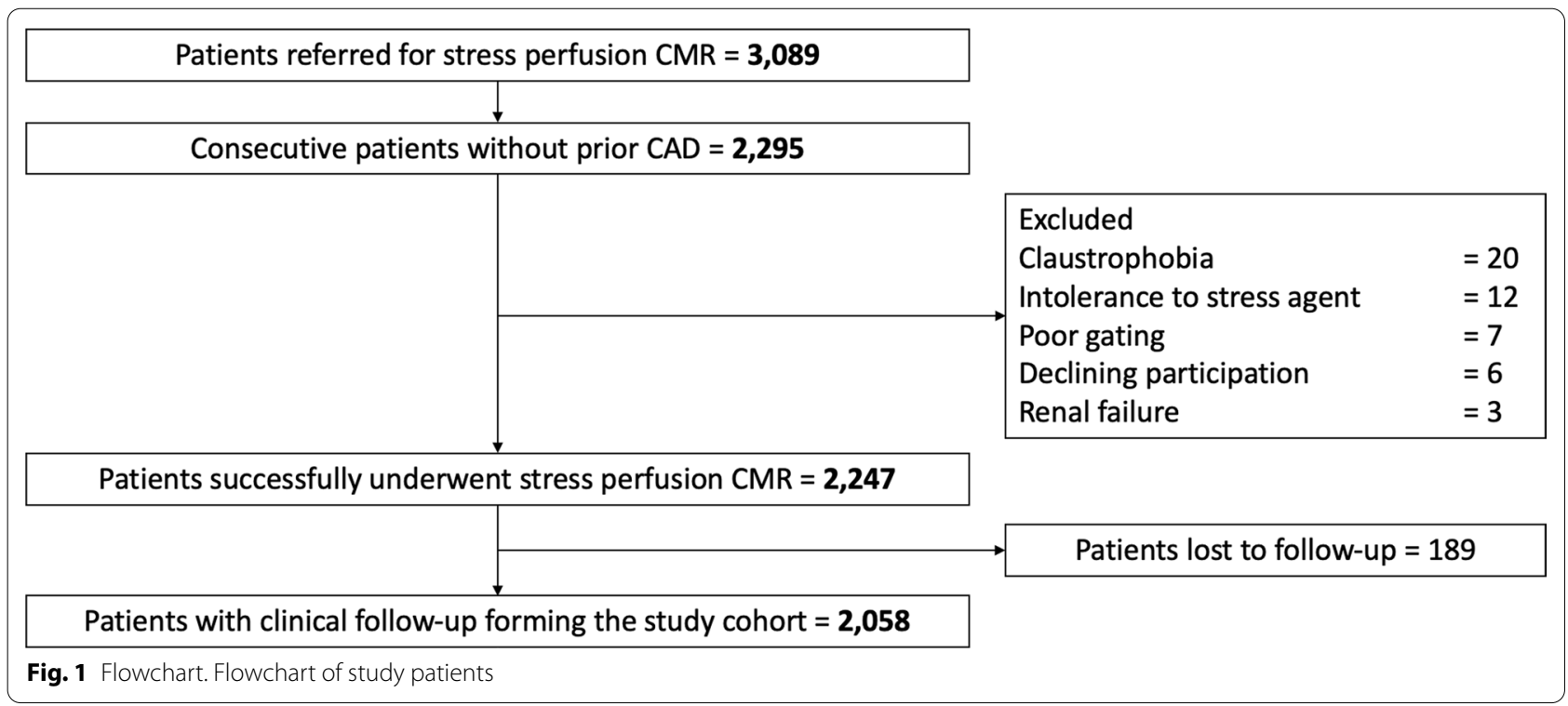




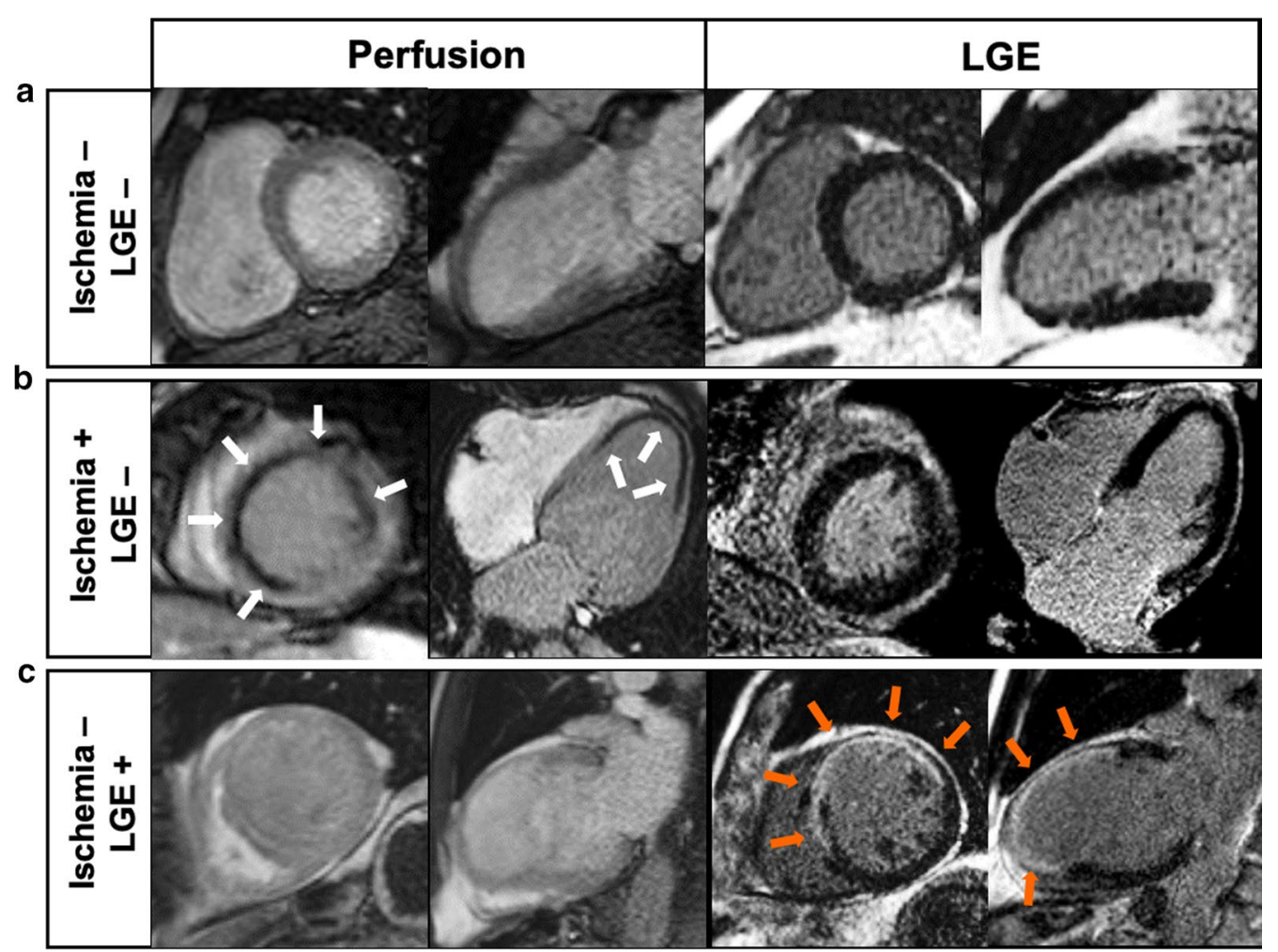

Fig. 2 Examples of inducible ischemia on stress CMR in patients without prior CAD. a fifty-three-year-old female without prior CAD but with diabetes, dyslipidemia and hypertension. Stress CMR revealed no perfusion defect and no LGE, excluding the diagnosis of CAD. $\mathbf{b}$ Fifty-eight-year-old female without prior CAD but with diabetes and obesity referred for atypical chest pain. Stress CMR showed a subendocardial perfusion defect on the anteroseptal wall on first-pass perfusion images (white arrows) without myocardial scar on LGE, indicative of myocardial ischemia. Coronary angiography revealed a high-grade stenosis of the LAD. c Seventy-one-year-old male without prior CAD but with diabetes, hypertension and heredity referred for atypical chest pain. Stress CMR showed a transmural anteroseptal MI on LGE (orange arrows) without perfusion defect, therefore no ischemia. Coronary angiography confirmed the chronic occlusion of the LAD and the absence of other significant stenosis. CAD coronary artery disease, $L A D$ left anterior descending, $L G E$ late gadolinium enhancement, $R C A$ right coronary artery

all significantly associated with MACE. Using KaplanMeier analysis, ischemia and unrecognized MI were associated with the occurrence of MACE (HR: $4.6495 \% \mathrm{CI}$ : 3.69-6.17 and HR: 2.88; 95\% CI: 2.08-3.99, respectively; both $\mathrm{p}<0.001$ ) (Fig. 3). In addition, ischemia was associated with cardiovascular mortality (HR: 4.00; 95\% CI: 2.85-5.61), non-fatal MI (HR: 6.20; 95\% CI: 3.61-10.70) and all-cause mortality (HR: 2.74 ; $95 \%$ CI: $2.11-3.55$, all $\mathrm{p}<0.001$; Additional file 1: Table S1). The presence of ischemia was significantly associated with MACE in men (HR: 5.29; 95\% CI: 3.57-7.84) and in women (HR: 3.33; 95\% CI: $2.08-5.34$, both $\mathrm{p}<0.001)$. The prognostic value of the presence of ischemia to predict MACE was significant in both asymptomatic and symptomatic patients (HR: 3.84; 95\% CI: 2.53-5.82 and HR: 5.55; 95\% CI: 3.748.24 , respectively, both $\mathrm{p}<0.001$ ).

The prognostic value of ischemia remained consistent in all other subsamples of clinical interest such as diabetics and non-diabetics, and regardless of LVEF (Fig. 4). In addition, the presence of ischemia had a similar prognostic value regardless of the age (Additional file 1: Figure S3).

In multivariable stepwise Cox regression (model 2), the presence of ischemia and unrecognized MI were independent predictors of MACE $(\mathrm{HR}=3.71 ; 95 \% \mathrm{CI}$ 2.73-5.05, $p<0.001$ and $\mathrm{HR}=1.73$; $95 \%$ CI $1.22-2.45$, $p=0.002$; respectively) and cardiovascular mortality (HR: 3.13; 95\% CI: $2.17-4.51, \mathrm{p}<0.001$ and $\mathrm{HR}=1.73$; $95 \% \mathrm{CI}$ $1.15-2.62, p=0.009$; respectively) (Table 3 ).

\section{Incremental prognostic value of stress CMR}

For the prediction of MACE, we observed baseline $\mathrm{C}$ statistic values of 0.61 (95\% CI, 0.56-0.65) for model 1 with stepwise variable selection. The addition of unrecognized MI significantly improved the C statistic to 0.66 (95\% CI, 0.60-0.71; C statistic improvement for model 1: 0.05; $\mathrm{NRI}=0.252$; IDI $=0.037$ ). Furthermore, the addition of unrecognized MI and ischemia significantly improved the C statistic to 0.72 (95\% CI, 0.69-0.76; C statistic 
Table 1 Baseline and CMR characteristics of patients with and without inducible ischemia $(\mathrm{N}=2058)$

\begin{tabular}{|c|c|c|c|c|}
\hline & $\begin{array}{l}\text { All patients } \\
(\mathrm{N}=2058)\end{array}$ & $\begin{array}{l}\text { No ischemia } \\
(\mathrm{N}=1791)\end{array}$ & $\begin{array}{l}\text { Positive ischemia } \\
(\mathrm{N}=267)\end{array}$ & $\mathrm{p}$ value \\
\hline Age, years & $71.2 \pm 12.5$ & $70.8 \pm 12.6$ & $74.7 \pm 11.4$ & $<0.001$ \\
\hline Males, n (\%) & $772(37.5)$ & $613(34.2)$ & $159(59.6)$ & $<0.001$ \\
\hline Body mass index, $\mathrm{kg} / \mathrm{m}^{2}$ & $29.4 \pm 6.6$ & $29.6 \pm 6.7$ & $28.3 \pm 5.7$ & $<0.001$ \\
\hline \multicolumn{5}{|l|}{ Coronary artery disease risk factors, $\mathrm{n}(\%)$} \\
\hline Diabetes mellitus & $702(34.1)$ & $618(34.5)$ & $84(31.5)$ & 0.363 \\
\hline Hypertension & $1358(66.0)$ & $1193(66.6)$ & $165(61.8)$ & 0.139 \\
\hline Dyslipidemia & $1007(48.9)$ & $875(48.9)$ & $132(49.4)$ & 0.911 \\
\hline Current or previous smoking & $436(21.2)$ & $378(21.1)$ & $58(21.7)$ & 0.881 \\
\hline Family history of coronary disease & $512(24.9)$ & $450(25.1)$ & $62(23.2)$ & 0.551 \\
\hline Obesity ${ }^{a}$ & $726(35.3)$ & $644(36.0)$ & $82(30.7)$ & 0.109 \\
\hline \multicolumn{5}{|l|}{ Medical history of cardiovascular disease, $n$ (\%) } \\
\hline Peripheral atheroma & $52(2.5)$ & $45(2.5)$ & $7(2.6)$ & 0.662 \\
\hline Ischemic stroke & $90(4.4)$ & $78(4.4)$ & $12(4.5)$ & 0.877 \\
\hline Pacemaker & $10(0.5)$ & $9(0.5)$ & $1(0.4)$ & 1.000 \\
\hline Renal failure ${ }^{\mathrm{b}}$ & $23(1.1)$ & $21(1.2)$ & $2(0.7)$ & 0.759 \\
\hline Prior hospitalization for heart failure & $49(2.4)$ & $43(2.4)$ & $6(2.3)$ & 1.000 \\
\hline \multicolumn{5}{|l|}{ Indications to stress CMR (multiple possible), n (\%) } \\
\hline High cardiovascular disease risk ${ }^{c}$ & $724(35.2)$ & $572(31.9)$ & $152(56.9)$ & $<0.001$ \\
\hline Symptomatic angina & $530(25.8)$ & $447(25.0)$ & $83(31.1)$ & 0.039 \\
\hline Dyspnea & $547(26.6)$ & $490(27.4)$ & $57(21.3)$ & 0.046 \\
\hline Inconclusive stress test & $410(19.9)$ & $360(20.1)$ & $50(18.7)$ & 0.658 \\
\hline Inconclusive coronary CT angiogram ${ }^{d}$ & $26(1.3)$ & $24(1.3)$ & $2(0.7)$ & 0.566 \\
\hline Ten-year risk for fatal CAD (\%) ${ }^{e}$ & $2.6(1.1-5.6)$ & $2.4(0.9-5.4)$ & $3.4(1.7-6.4)$ & $<0.001$ \\
\hline \multicolumn{5}{|l|}{ Cardiac rhythm, n (\%) } \\
\hline Sinus rhythm & $1507(73.2)$ & $1288(71.9)$ & $219(82.0)$ & $<0.001$ \\
\hline Sinus rhythm with extrasystoles & $542(26.3)$ & $495(27.6)$ & $47(17.6)$ & \\
\hline Atrial fibrillation/supraventricular arrhythmias & $9(0.4)$ & $8(0.4)$ & $1(0.4)$ & \\
\hline LV ejection fraction, \% & $52.6 \pm 12.7$ & $52.9 \pm 12.7$ & $50.6 \pm 12.7$ & 0.007 \\
\hline LV end-diastolic volume index, $\mathrm{ml} / \mathrm{m}^{2}$ & $81.1 \pm 27.5$ & $81.1 \pm 27.3$ & $81.5 \pm 29.5$ & 0.844 \\
\hline LV end-systolic volume index, $\mathrm{ml} / \mathrm{m}^{2}$ & $40.6 \pm 23.4$ & $40.3 \pm 23.3$ & $42.3 \pm 24.4$ & 0.208 \\
\hline LV mass, $\mathrm{g} / \mathrm{m}^{2}$ & $75.5 \pm 7.7$ & $72.9 \pm 7.7$ & $77.9 \pm 7.8$ & $<0.001$ \\
\hline RV ejection fraction, \% & $65.9 \pm 12.3$ & $65.9 \pm 12.3$ & $65.8 \pm 12.5$ & 0.831 \\
\hline Presence of unrecognized Ml, $\mathrm{n}(\%)$ & $210(10.2)$ & $135(7.5)$ & $75(28.1)$ & $<0.001$ \\
\hline Number of segments of LGE & $0.3 \pm 1.0$ & $0.2 \pm 0.9$ & $0.8 \pm 1.6$ & $<0.001$ \\
\hline Number of segments of ischemia & $0.3 \pm 1.1$ & $0.0 \pm 0.0$ & $2.6 \pm 1.7$ & $<0.001$ \\
\hline Heart rate at baseline, beats/min & $80 \pm 13$ & $80 \pm 13$ & $81 \pm 15$ & 0.688 \\
\hline Heart rate at stress, beats/min & $92 \pm 12$ & $92 \pm 11$ & $94 \pm 13$ & 0.569 \\
\hline RPP at baseline, $\mathrm{mmHg} /$ beats/min & $9.1(7.6-10.8)$ & $9.1(7.6-10.7)$ & $9.2(7.6-10.9)$ & 0.733 \\
\hline RPP at stress, mmHg/beats/min & $10.4(8.8-12.6)$ & $10.4(8.8-12.2)$ & $11.2(9.8-13.3)$ & 0.363 \\
\hline
\end{tabular}

Values are $\mathrm{n}(\%)$, mean $\pm \mathrm{SD}$, or median (interquartile range)

$B M I$ body mass index, $C A D$ coronary artery disease, $C T A$ computed tomography, $C M R$ cardiovascular magnetic resonance, $L G E$ late gadolinium enhancement, $L V$ left ventricle, $M I$ myocardial infarction, $R P P$ rate-pressure product (pressure $\mathrm{mmHg} \times$ Heart rate bpm)/1000, $R V$ right ventricle, $S D$ standard deviation

a Defined by body mass index $\geq 30 \mathrm{~kg} / \mathrm{m}^{2}$

b Defined by estimated glomerular filtration rate $<60 \mathrm{ml} / \mathrm{min} / 1.73 \mathrm{~m}^{2}$

c Defined by Framingham Risk Score $>20 \%$ of risk of CAD at 10 years

d Defined by coronary stenosis of unknown significance on coronary CT angiography 


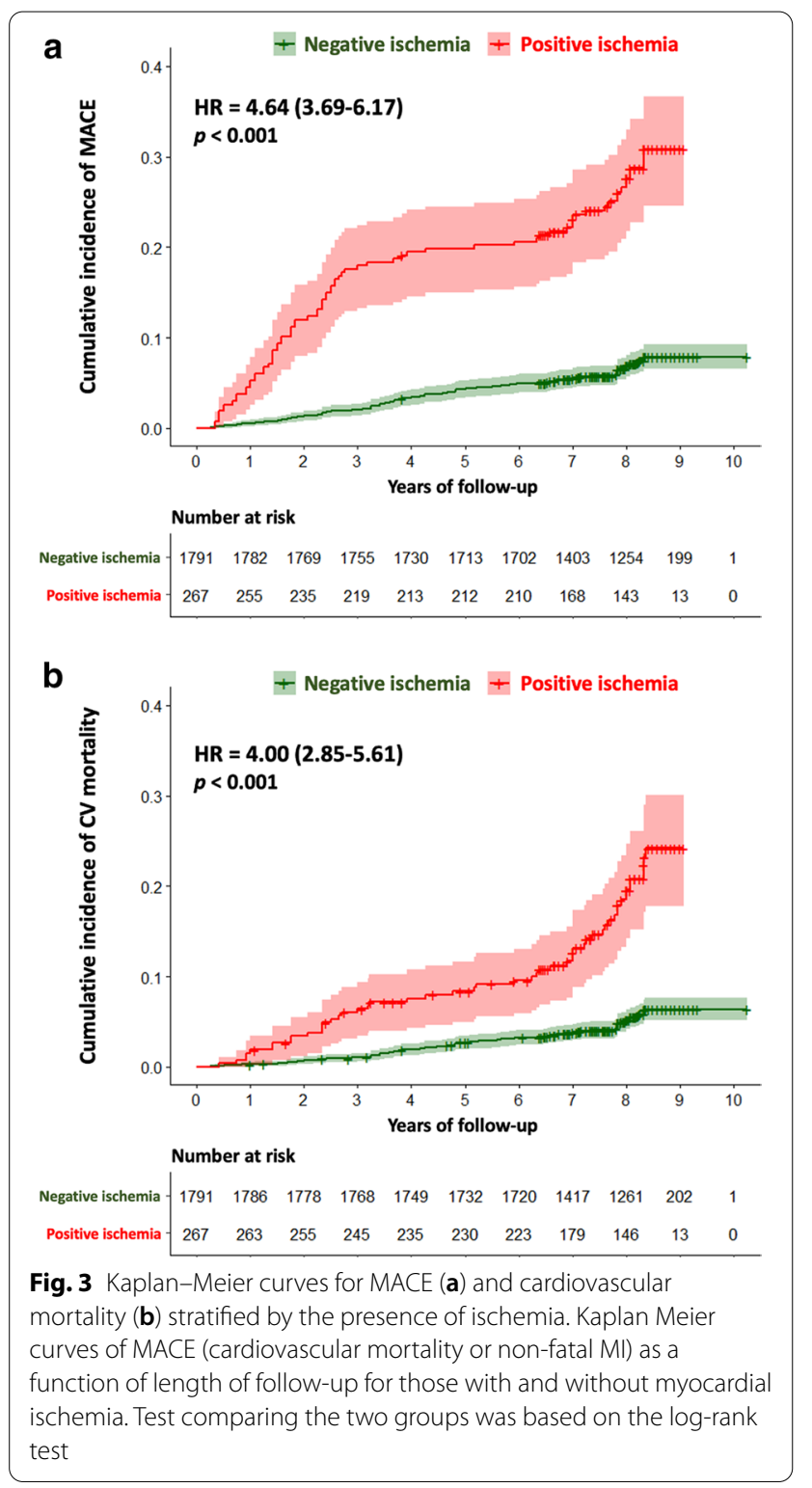

improvement for model 1: 0.11 ;RI $=0.431 ; \mathrm{IDI}=0.053$ ) (Table 4).

\section{Discussion}

In this large single center study of patients without known CAD referred for stress CMR, our main findings demonstrate that: (1) inducible ischemia and unrecognized MI were independent long-term predictors of MACE and cardiovascular mortality; (2) the presence of inducible ischemia and unrecognized MI improved model discrimination for the prediction of MACE, after adjusting for covariates; (3) $13.0 \%$ of patients had inducible ischemia and $10.2 \%$ had unrecognized MI.
Previous studies in patients with suspected or known CAD reported that the prevalence of CMR-inducible ischemia ranged between 7 and $26 \%[4,6,15-17]$. In the current study including low-risk patients without known CAD, the prevalence of inducible ischemia was $13.0 \%$. The prevalence of unrecognized MI detected by CMR has been shown to range between 0.2 and $30 \%$ in the general population $[9,31,32]$, about $15 \%$ in symptomatic patients [11], and less than $6 \%$ in asymptomatic patients with suspected CAD [17]. In the present study, the prevalence of unrecognized MI was $10.2 \%$. Consistently with prior cohorts of patients without known CAD $[4,13,15-17]$, the rate of MACE was $9.9 \%$ over a median follow-up of 8.3 years.

Stress-CMR inducible ischemia and unrecognized MI were independently associated with MACE in the sole subset of patients without known CAD. Such findings extend the aggregate data on the prognostic value of stress CMR [4, 6, 11, 15, 16, 33, 34]. Consistently, the extent of ischemia was a strong prognosticator of MACE and cardiovascular mortality, as already described in patients with known or suspected CAD [13]. Several studies have shown similar accuracy to diagnose $\mathrm{CAD}$ and predict cardiovascular events in men and women $[35,36]$. In line with these data, the current study suggests a similar prognostic value of stress CMR in women and men.

Although some studies have suggested the prognostic value of stress CMR in patients without known $C A D$ $[5,15-17]$, they have not evaluated the incremental prognostic value of stress CMR to predict cardiovascular events over traditional risk factors in this population. The current study demonstrates an incremental prognostic value of unrecognized MI to predict MACE above traditional cardiovascular risk factors and LVEF. This finding is in line with the recent SPINS registry of the Society for Cardiovascular Magnetic Resonance showing that presence of unrecognized MI portended a significant risk for cardiovascular events, independently of the presence of ischemia [11]. In the current, the addition of inducible ischemia to the model containing traditional risk factors and unrecognized MI, further improved the prognostic value for predicting MACE. These data highlight the importance of integrating both inducible ischemia and unrecognized MI in risk stratification models.

This addition of unrecognized MI and ischemia by stress CMR in risk stratification models led to an incremental prognostic value, as illustrated by a rise in the C-index from 0.61 to 0.66 and 0.72 , respectively. Whereas this prognostic incremental value of stress CMR could be cost-effective remains to be evaluated. The SPINS registry [4] demonstrated that the average cost of ischemic 


\begin{tabular}{|c|c|c|c|c|c|c|}
\hline Group / Subgroup & $\begin{array}{l}\text { Ischemia } \\
\text { N events/N } \\
\text { subgroup* }\end{array}$ & $\begin{array}{l}\text { No Ischemia } \\
\mathrm{N} \text { events/N } \\
\text { subgroup* }\end{array}$ & & $\begin{array}{c}\text { Hazard ratio } \\
(95 \% \text { CI })\end{array}$ & $p$ value & $\begin{array}{c}p \text { value } \\
\quad \text { for } \\
\text { interaction }\end{array}$ \\
\hline Age & & & & & & 0.088 \\
\hline$<65$ years & $15 / 51$ & $26 / 545$ & $\longmapsto$ & $7.29(3.86-13.80)$ & $<0.001$ & \\
\hline$\geq 65$ years & $60 / 216$ & $02 / 1246$ & $\longmapsto$ & $3.99(2.90-5.49)$ & $<0.001$ & \\
\hline Sex & & & & & & 0.136 \\
\hline Female & $22 / 108$ & $81 / 1178$ & $\longmapsto$ & $3.33(2.08-5.34)$ & $<0.001$ & \\
\hline Male & $53 / 159$ & $47 / 613$ & $\longmapsto$ & $5.29(3.57-7.84)$ & $<0.001$ & \\
\hline Body Mass Index & & & & & & 0.071 \\
\hline No obesity $\left(\mathrm{BMI}<30 \mathrm{~kg} / \mathrm{m}^{2}\right)$ & $47 / 185$ & $87 / 1147$ & $\longmapsto$ & $3.87(2.71-5.52)$ & $<0.001$ & \\
\hline Obesity (BMI $\geq 30 \mathrm{~kg} / \mathrm{m}^{2}$ ) & $28 / 82$ & $41 / 644$ & $\longmapsto$ & $6.60(4.08-10.70)$ & $<0.001$ & \\
\hline Diabetes melitus & & & & & & 0.709 \\
\hline No & $52 / 183$ & $88 / 1173$ & $\longmapsto$ & $4.46(3.17-6.29)$ & $<0.001$ & \\
\hline Yes & $23 / 84$ & $40 / 618$ & $\longmapsto$ & $4.99(2.99-8.34)$ & $<0.001$ & \\
\hline Hypertension & & & & & & 0.514 \\
\hline No & 29/102 & $48 / 598$ & $\longmapsto$ & $4.05(2.56-6.43)$ & $<0.001$ & \\
\hline Yes & $46 / 165$ & $80 / 1193$ & $\longmapsto$ & $5.01(3.48-7.20)$ & $<0.001$ & \\
\hline Smoking & & & & & & 0.580 \\
\hline No & $60 / 209$ & $101 / 1413$ & $\longmapsto$ & $4.86(3.53-6.69)$ & $<0.001$ & \\
\hline Yes & $15 / 58$ & $27 / 378$ & $\longmapsto$ & $3.92(2.09-7.37)$ & $<0.001$ & \\
\hline LVEF & & & & & & 0.660 \\
\hline$<50 \%$ & $28 / 99$ & $44 / 556$ & $\longmapsto$ & $4.16(2.59-6.68)$ & $<0.001$ & \\
\hline$>=50 \%$ & $47 / 168$ & $84 / 1235$ & $\longmapsto$ & $4.87(3.41-6.96)$ & $<0.001$ & \\
\hline
\end{tabular}

Fig. 4 Subgroup analysis. Forest-plot of incidence of MACE based on the presence of ischemia in prespecified subgroups. ${ }^{*} \mathrm{~N}$ events/ $\mathrm{N}$ subgroup: number of patients who had a major adverse clinical event (MACE) / number of patients in the subgroup

Table 2 Univariable analysis of clinical and CMR characteristics for prediction of adverse events

\begin{tabular}{|c|c|c|c|c|}
\hline & \multicolumn{2}{|l|}{ MACE } & \multicolumn{2}{|c|}{ Cardiovascular mortality } \\
\hline & Hazard ratio $(95 \% \mathrm{Cl})$ & $p$ value & Hazard ratio $(95 \% \mathrm{Cl})$ & p value \\
\hline Age & $1.02(1.01-1.03)$ & 0.002 & $1.03(1.02-1.05)$ & $<0.001$ \\
\hline Male & $1.68(1.27-2.21)$ & $<0.001$ & $1.60(1.16-2.20)$ & 0.004 \\
\hline Body mass index & $0.98(0.95-1.02)$ & 0.311 & $1.01(0.95-1.03)$ & 0.485 \\
\hline Hypertension & $0.83(0.62-1.10)$ & 0.190 & $0.88(0.63-1.22)$ & 0.431 \\
\hline Diabetes mellitus & $0.86(0.64-1.15)$ & 0.307 & $0.87(0.62-1.23)$ & 0.429 \\
\hline Dyslipidemia & $1.00(0.76-1.32)$ & 0.978 & $1.00(0.73-1.38)$ & 0.978 \\
\hline Current or previous smoking & $0.96(0.68-1.35)$ & 0.811 & $0.81(0.53-1.22)$ & 0.309 \\
\hline Family history of coronary artery disease & $0.72(0.51-1.02)$ & 0.064 & $0.61(0.40-0.94)$ & 0.023 \\
\hline Stroke & $0.77(0.37-1.83)$ & 0.371 & $0.74(0.30-1.19)$ & 0.466 \\
\hline Renal failure & $1.36(0.44-4.26)$ & 0.595 & $1.27(0.31-5.12)$ & 0.738 \\
\hline Peripheral atheroma & $1.12(0.29-3.37)$ & 0.566 & $1.54(0.34-4.45)$ & 0.319 \\
\hline Prior hospitalization for heart failure & $1.57(0.74-3.34)$ & 0.240 & $1.87(0.83-4.24)$ & 0.133 \\
\hline Presence of ischemia & $4.64(3.49-6.17)$ & $<0.001$ & $4.00(2.85-5.61)$ & $<0.001$ \\
\hline Number of segments of ischemia & $1.60(1.51-1.70)$ & $<0.001$ & $1.51(1.40-1.62)$ & $<0.001$ \\
\hline Presence of unrecognized Ml & $2.88(2.08-3.99)$ & $<0.001$ & $2.77(1.89-4.06)$ & $<0.001$ \\
\hline Number of segments of LGE & $1.52(1.41-1.63)$ & $<0.001$ & $1.49(1.37-1.62)$ & $<0.001$ \\
\hline LV ejection fraction, per $10 \%$ & $0.88(0.79-0.97)$ & 0.014 & $0.93(0.82-1.05)$ & 0.245 \\
\hline LV end-diastolic volume index, per $10 \mathrm{ml} / \mathrm{m}^{2}$ & $1.06(1.01-1.11)$ & 0.025 & $1.03(0.97-1.09)$ & 0.348 \\
\hline LV end-systolic volume index, per $10 \mathrm{ml} / \mathrm{m}^{2}$ & $1.06(1.02-1.13)$ & 0.009 & $1.04(0.97-1.11)$ & 0.252 \\
\hline RV ejection fraction, \% & $0.96(0.79-1.19)$ & 0.411 & $1.06(0.78-1.53)$ & 0.681 \\
\hline
\end{tabular}

$C I$ confidence interval, $C M R$ cardiovascular magnetic resonance, $L G E$ late gadolinium enhancement, $L V$ left ventricle, $M A C E$ major adverse cardiac events, $M I$ myocardial infarction, $R V$ right ventricle 
Table 3 Multivariable cox regression analysis for the prediction of adverse events

\begin{tabular}{|c|c|c|c|c|}
\hline & \multicolumn{2}{|l|}{ MACE } & \multicolumn{2}{|c|}{ Cardiovascular mortality } \\
\hline & $\begin{array}{l}\text { Hazard ratio } \\
(95 \% \mathrm{Cl})\end{array}$ & $p$ value & $\begin{array}{l}\text { Hazard ratio } \\
(95 \% \mathrm{Cl})\end{array}$ & $p$ value \\
\hline \multicolumn{5}{|l|}{ Model $1^{a}$} \\
\hline Age & $1.03(1.01-1.05)$ & 0.002 & $1.04(1.02-1.07)$ & $<0.001$ \\
\hline Male & $1.91(1.32-2.76)$ & $<0.001$ & $1.85(1.22-2.82)$ & 0.004 \\
\hline Family history of coronary artery disease & $0.74(0.49-1.10)$ & 0.131 & $0.66(0.41-1.05)$ & 0.076 \\
\hline LV end-systolic volume index, per $10 \mathrm{ml} / \mathrm{m}^{2}$ & $2.70(0.99-7.37)$ & 0.053 & $3.81(1.38-10.5)$ & 0.010 \\
\hline \multicolumn{5}{|l|}{ Model $2^{b}$} \\
\hline Presence of unrecognized Ml & $1.82(1.28-2.49)$ & $<0.001$ & $1.76(1.18-2.66)$ & 0.007 \\
\hline \multicolumn{5}{|l|}{ Model $3^{c}$} \\
\hline Presence of unrecognized MI & $1.73(1.22-2.45)$ & 0.002 & $1.73(1.15-2.62)$ & 0.009 \\
\hline Presence of ischemia & $3.71(2.73-5.05)$ & $<0.001$ & $3.13(2.17-4.51)$ & $<0.001$ \\
\hline
\end{tabular}

Table 4 Discrimination and reclassification associated with ischemia and LGE for prediction of MACE

\begin{tabular}{|c|c|c|c|}
\hline & \multicolumn{3}{|l|}{ MACE } \\
\hline & C-index $(95 \% \mathrm{Cl})$ & $\mathrm{NRI}(95 \% \mathrm{Cl})$ & IDI (95\%Cl) \\
\hline Model 1 (stepwise selection) ${ }^{a}$ & $0.61(0.56-0.65)$ & Reference & Reference \\
\hline Model 2 (model $1+$ unrecognized MI) ${ }^{b}$ & $0.66(0.60-0.71)$ & $0.252(0.065-0.439)$ & $0.037(0.016-0.058)$ \\
\hline Model 3 (model 1 + unrecognized MI and ischemia) ${ }^{c}$ & $0.72(0.69-0.76)$ & $0.431(0.212-0.650)$ & $0.053(0.030-0.076)$ \\
\hline \multicolumn{4}{|c|}{$\mathrm{Cl}$ confidence interval, IDI integrative discrimination index, $M A C E$ major adverse cardiac events, $M I$ myocardial infarction, $N R I$ net reclassification improvement } \\
\hline \multicolumn{4}{|c|}{$\begin{array}{l}\text { a Covariates in the model } 1 \text { by stepwise variable selection with entry and exit criteria set at the } p \leq 0.2 \text { level: age, male, hypertension, family history of coronary artery } \\
\text { disease, LVEF per } 10 \% \text {, LV end-systolic volume index, per } 10 \mathrm{ml} / \mathrm{m}^{2}\end{array}$} \\
\hline \multicolumn{4}{|l|}{ b Covariates in the model 2: model 1 with unrecognized MI } \\
\hline \multicolumn{4}{|c|}{ c Covariates in the model 3: model 1 with unrecognized $\mathrm{Ml}$ and ischemia } \\
\hline
\end{tabular}

testing was lower for stress CMR than other stress testing [37]. If cost-effective, the current data support the use of stress CMR to identify high-risk patients who could benefit from improved clinical and therapeutic management $[38,39]$.

\section{Study limitations}

Our study has several limitations. First, the study was retrospective, with a risk of referral bias. Overall, 189 (8.4\%) patients were lost to follow-up, which can be explained by a relatively long follow-up and the design of the study. However, the French National Registry of Death was carefully reviewed, which strengthens the data on mortality. Despite the good prognostic value of stress CMR in AF patients [40], the low proportion of AF patients referred for stress CMR is likely due to the reluctance of referring cardiologists. This study was not designed to compare the prognostic value in women and men. The analysis of CMR stress perfusion images was visual, which represents the most widely accepted clinical method with optimal diagnostic accuracy. Stress perfusion CMR protocol included six slices (4 short-axis views, and long-axis views) acquired every other heartbeat to optimize anatomical coverage of the LV, at the cost of a slight decrease in temporal resolution. This retrospective study could not capture all of the confounding factors regarding the association between management decisions after the stress CMR exam and patient risk. Finally, the extent of myocardial scar was assessed semi-quantitatively by the number of infarcted segments and not quantitatively by semiautomated methods. 


\section{Conclusions}

Stress perfusion CMR has a good discriminative longterm prognostic value in patients without known CAD. Stress-CMR inducible ischemia and unrecognized MI are independently associated with non-fatal MI and cardiovascular mortality over a long-term follow-up and offer incremental prognostic value over traditional risk factors.

\section{Author details}

${ }^{1}$ CMR Department, Institut Cardiovasculaire Paris Sud, Cardiovascular Magnetic Resonance Laboratory, Hôpital Privé Jacques CARTIER, Ramsay Santé, 6 Avenue du Noyer Lambert, 91300 Massy, France. ${ }^{2}$ Division of Cardiology, Johns Hopkins University, Baltimore, MD 21287-0409, USA. ${ }^{3}$ Siemens Healthcare France, 93200 Saint-Denis, France.

Received: 23 September 2020 Accepted: 21 February 2021

Published online: 08 April 2021

\begin{abstract}
Abbreviations
BMI: Body mass index; BNP: Brain natriuretic peptide; bSSFP: Balanced steady state free precession; $C A B G$ : Coronary artery bypass graft; $C A D$ : Coronary artery disease; $\mathrm{Cl}$ : Confidence interval; CMR: Cardiovascular magnetic resonance; ECG: Electrocardiogram; FFR: Fractional flow reserve; HR: Hazard ratio; IDI: Integrative discrimination index; LGE: Late gadolinium enhancement; LV: Left ventricle/left ventricular; LVEF: Left ventricular ejection fraction; MACE: Major adverse cardiac event; MI: Myocardial infarction; NRI: Net reclassification improvement; PCI: Percutaneous coronary intervention; SD: Standard deviation; IQR: Interquartile range.
\end{abstract}

\section{Supplementary Information}

The online version contains supplementary material available at https://doi. org/10.1186/s12968-021-00737-0.

Additional file 1: Figure S1. The assessment of the proportional hazard assumption. Figure S2. Annualized event rates of MACE (A) and cardiovascular mortality (B) stratified by the extent of ischemia ( $N=2058)$. Table S1. Univariable analysis of inducible myocardial ischemia for prediction of adverse events $(N=2058)$. Figure S3. Annualized event rates of MACE stratified by age and presence/absence of ischemia.

\section{Acknowledgements}

We thank the medical, paramedical and research staff of Hôpital Privé J. CARTIER.

\section{Authors' contributions}

All authors participated in the discussion of the concept of the study. TP and JG conceived the study design. TP, FS, MK, TH, SC, TU, PG and JG, obtained CMR images and analyzed CMR scans. TP and JG analyzed data and drafted the manuscript with critical revision. JG and ST have technically defined the CMR protocol. As authors, we attest to each of our substantial contributions to the manuscript and revision. All authors read and approved the final manuscript.

\section{Funding}

None.

\section{Availability of data and materials}

All data generated or analysed during this study are included in this published article [and its Additional information files].

\section{Ethics approval and consent to participate}

The study was evaluated and approved by the local ethic committee of our institutions and conducted in accordance with the 1964 Declaration of Helsinki. All patients enrolled in this study were required to understand and give their consent for participation.

\section{Consent for publication}

Not applicable.

\section{Competing interests}

Solenn Toupin is an employee of Siemens Healthcare. Other authors declare that they have no competing interests.

\section{References}

1. Benjamin EJ, Muntner P, Alonso A, Bittencourt MS, Callaway CW, Carson AP, et al. Heart disease and stroke statistics - 2019 update: a report from the American Heart Association. Circulation. 2019;139(10):e56-528.

2. Knuuti J, Wijns W, Saraste A, Capodanno D, Barbato E, Funck-Brentano C, et al. 2019 ESC Guidelines for the diagnosis and management of chronic coronary syndromes. Eur Heart J. 2020;41(3):407-77.

3. Yancy CW, Jessup M, Bozkurt B, Butler J, Casey DE, Drazner MH, et al. 2013 ACCF/AHA guideline for the management of heart failure: a report of the American College of Cardiology Foundation/American Heart Association Task Force on Practice Guidelines. J Am Coll Cardiol. 2013;62(16):e147-239.

4. Kwong RY, GeY, Steel K, Bingham S, Abdullah S, Fujikura K, et al. Cardiac magnetic resonance stress perfusion imaging for evaluation of patients with chest pain. J Am Coll Cardiol. 2019;74(14):1741-55.

5. Shah R, Heydari B, Coelho-Filho O, Murthy VL, Abbasi S, Feng JH, et al. Stress cardiac magnetic resonance imaging provides effective cardiac risk reclassification in patients with known or suspected stable coronary artery disease. Circulation. 2013;128(6):605-14.

6. Heitner JF, Kim RJ, Kim HW, Klem I, Shah DJ, Debs D, et al. Prognostic value of vasodilator stress cardiac magnetic resonance imaging: a multicenter study with 48000 patient-years of follow-up. JAMA Cardiol. 2019:4(3):256-64.

7. Antiochos P, Ge Y, Steel K, Chen Y-Y, Bingham S, Abdullah S, et al. Evaluation of stress cardiac magnetic resonance imaging in risk reclassification of patients with suspected coronary artery disease. JAMA Cardiol. 2020;5(12):1401.

8. Acharya T, Aspelund T, Jonasson TF, Schelbert EB, Cao JJ, Sathya B, et al. Association of unrecognized myocardial infarction with long-term outcomes in community-dwelling older adults: the ICELAND MI Study. JAMA Cardiol. 2018;3(11):1101-6.

9. Schelbert EB, Cao JJ, Sigurdsson S, Aspelund T, Kellman P, Aletras AH, et al. Prevalence and prognosis of unrecognized myocardial infarction determined by cardiac magnetic resonance in older adults. JAMA. 2012;308(9):890-6.

10. Kwong RY, Sattar H, Wu H, Vorobiof G, Gandla V, Steel K, et al. Incidence and prognostic implication of unrecognized myocardial scar characterized by cardiac magnetic resonance in diabetic patients without clinical evidence of myocardial infarction. Circulation. 2008;118(10):1011-20.

11. Antiochos P, Ge Y, Steel K, Bingham S, Abdullah S, Mikolich JR, et al. Imaging of clinically unrecognized myocardial fibrosis in patients with suspected coronary artery disease. J Am Coll Cardiol. 2020;76(8):945-57.

12. Nagel E, Greenwood JP, McCann GP, Bettencourt N, Shah AM, Hussain ST, et al. Magnetic resonance perfusion or fractional flow reserve in coronary disease. N Engl J Med. 2019;380(25):2418-28.

13. Vincenti G, Masci PG, Monney P, Rutz T, Hugelshofer S, Gaxherri M, et al. Stress perfusion CMR in patients with known and suspected CAD. JACC Cardiovasc Imaging. 2017;10(5):526-37.

14. Greenwood JP, Herzog BA, Brown JM, Everett CC, Nixon J, Bijsterveld P, et al. Prognostic value of cardiovascular magnetic resonance and singlephoton emission computed tomography in suspected coronary heart disease: long-term follow-up of a prospective, diagnostic accuracy cohort study. Ann Intern Med. 2016;165(1):1-9.

15. Kinnel M, Garot J, Pezel T, Hovasse T, Unterseeh T, Champagne S, et al. Prognostic value of vasodilator stress perfusion CMR in morbidly obese patients (BMI $\geq 40 \mathrm{~kg} / \mathrm{m}^{2}$ ) without known CAD. JACC Cardiovasc Imaging. 2020;13(5):1276-7.

16. Pezel T, Sanguineti F, Kinnel M, Hovasse T, Garot P, Unterseeh T, et al. Prognostic value of dipyridamole stress perfusion cardiovascular magnetic 
resonance in elderly patients $>75$ years with suspected coronary artery disease. Eur Heart J Cardiovasc Imaging. 2020;jeaa193.

17. Stacey RB, Vera T, Morgan TM, Jordan JH, Whitlock MC, Hall ME, et al. Asymptomatic myocardial ischemia forecasts adverse events in cardiovascular magnetic resonance dobutamine stress testing of high-risk middle-aged and elderly individuals. J Cardiovasc Magn Reson. 2018;20(1):75.

18. Thygesen K, Alpert JS, Jaffe AS, Chaitman BR, Bax JJ, Morrow DA, et al. Fourth universal definition of myocardial infarction (2018). J Am Coll Cardiol. 2018;72(18):2231-64.

19. Hicks KA, Tcheng JE, Bozkurt B, Chaitman BR, Cutlip DE, Farb A, et al. 2014 ACC/AHA key data elements and definitions for cardiovascular endpoint events in clinical trials. J Am Coll Cardiol. 2015;66(4):403-69.

20. Ponikowski P, Voors AA, Anker SD, Bueno H, Cleland JGF, Coats AJS, et al. 2016 ESC Guidelines for the diagnosis and treatment of acute and chronic heart failure: The Task Force for the diagnosis and treatment of acute and chronic heart failure of the European Society of Cardiology (ESC) Developed with the special contribution of the Heart Failure Association (HFA) of the ESC. Eur Heart J. 2016;37(27):2129-200.

21. Stone GW, Ben-Yehuda O, Sabik JF, Kappetein AP, Serruys PW. Considerations for an optimal definition of procedural myocardial infarction. Eur Heart J. 2020;41(17):1704-5.

22. Cerqueira MD, Weissman NJ, Dilsizian V, Jacobs AK, Kaul S, Laskey WK, et al. Standardized myocardial segmentation and nomenclature for tomographic imaging of the heart. A statement for healthcare professionals from the Cardiac Imaging Committee of the Council on Clinical Cardiology of the American Heart Association. Circulation. 2002;105(4):539-42.

23. Schwitter J, Wacker CM, van Rossum AC, Lombardi M, Al-Saadi N, Ahlstrom $\mathrm{H}$, et al. MR-IMPACT: comparison of perfusion-cardiac magnetic resonance with single-photon emission computed tomography for the detection of coronary artery disease in a multicentre, multivendor, randomized trial. Eur Heart J. 2008;29(4):480-9.

24. Schwitter J, Wacker CM, Wilke N, Al-Saadi N, Sauer E, Huettle K, et al. MR-IMPACT II: Magnetic Resonance Imaging for Myocardial Perfusion Assessment in Coronary artery disease Trial: perfusion-cardiac magnetic resonance vs. single-photon emission computed tomography for the detection of coronary artery disease: a comparative multicentre, multivendor trial. Eur Heart J. 2013;34(10):775-81.

25. Plein S, Kozerke S, Suerder D, Luescher TF, Greenwood JP, Boesiger $P$, et al. High spatial resolution myocardial perfusion cardiac magnetic resonance for the detection of coronary artery disease. Eur Heart J. 2008;29(17):2148-55.

26. Mahrholdt H, Wagner A, Judd RM, Sechtem U, Kim RJ. Delayed enhancement cardiovascular magnetic resonance assessment of non-ischaemic cardiomyopathies. Eur Heart J. 2005;26(15):1461-74.

27. Pencina MJ, D'Agostino RB, D'Agostino RB, Vasan RS. Evaluating the added predictive ability of a new marker: From area under the ROC curve to reclassification and beyond. Stat Med. 2008;27(2):157-72.

28. Uno H, Tian L, Cai T, Kohane IS, Wei LJ. A unified inference procedure for a class of measures to assess improvement in risk prediction systems with survival data. Stat Med. 2013;32(14):2430-42.
29. Conroy RM, Pyörälä K, Fitzgerald AP, Sans S, Menotti A, De Backer G, et al. Estimation of ten-year risk of fatal cardiovascular disease in Europe: the SCORE project. Eur Heart J. 2003;24(11):987-1003.

30. Wilson PW, D'Agostino RB, Levy D, Belanger AM, Silbershatz H, Kannel WB. Prediction of coronary heart disease using risk factor categories. Circulation. 1998;97(18):1837-47.

31. Barbier CE, Nylander $R$, Themudo R, Ahlström H, Lind L, Larsson E-M, et al. Prevalence of unrecognized myocardial infarction detected with magnetic resonance imaging and its relationship to cerebral ischemic lesions in both sexes. J Am Coll Cardiol. 2011;58(13):1372-7.

32. Kwong RY, Chan AK, Brown KA, Chan CW, Reynolds HG, Tsang S, et al. Impact of unrecognized myocardial scar detected by cardiac magnetic resonance imaging on event-free survival in patients presenting with signs or symptoms of coronary artery disease. Circulation. 2006;113(23):2733-43.

33. Lipinski MJ, MCVey CM, Berger JS, Kramer CM, Salerno M. Prognostic value of stress cardiac magnetic resonance imaging in patients with known or suspected coronary artery disease: a systematic review and meta-analysis. J Am Coll Cardiol. 2013;62(9):826-38.

34. Kelle S, Chiribiri A, Vierecke J, Egnell C, Hamdan A, Jahnke C, et al. Longterm prognostic value of dobutamine stress CMR. JACC Cardiovasc Imaging. 2011;4(2):161-72.

35. Greenwood JP, Motwani M, Maredia N, Brown JM, Everett CC, Nixon J, et al. Comparison of cardiovascular magnetic resonance and singlephoton emission computed tomography in women with suspected coronary artery disease from the Clinical Evaluation of Magnetic Resonance Imaging in Coronary Heart Disease (CE-MARC) Trial. Circulation. 2014;129(10):1129-38.

36. Coelho-Filho OR, Seabra LF, Mongeon F-P, Abdullah SM, Francis SA, Blankstein R, et al. Stress myocardial perfusion imaging by CMR provides strong prognostic value to cardiac events regardless of patient's sex. JACC Cardiovasc Imaging. 2011;4(8):850-61.

37. Ge Y, Pandya A, Steel K, Bingham S, Jerosch-Herold M, Chen Y-Y, et al. Cost-effectiveness analysis of stress cardiovascular magnetic resonance imaging for stable chest pain syndromes. JACC Cardiovasc Imaging. 2020;13(7):1505-17.

38. Ridker PM, Everett BM, Thuren T, MacFadyen JG, Chang WH, Ballantyne $C$, et al. Antiinflammatory therapy with canakinumab for atherosclerotic disease. N Engl J Med. 2017;377(12):1119-31.

39. Eikelboom JW, Connolly SJ, Bosch J, Dagenais GR, Hart RG, Shestakovska $\mathrm{O}$, et al. Rivaroxaban with or without aspirin in stable cardiovascular disease. N Engl J Med. 2017;377(14):1319-30.

40. Pezel T, Sanguineti F, Kinnel M, Landon V, Toupin S, Unterseeh T, et al. Feasibility and prognostic value of vasodilator stress perfusion CMR in patients with atrial fibrillation. JACC Cardiovasc Imaging. 2021;14(2):379-89.

\section{Publisher's Note}

Springer Nature remains neutral with regard to jurisdictional claims in published maps and institutional affiliations.

\footnotetext{
Ready to submit your research? Choose BMC and benefit from:

- fast, convenient online submission

- thorough peer review by experienced researchers in your field

- rapid publication on acceptance

- support for research data, including large and complex data types

- gold Open Access which fosters wider collaboration and increased citations

- maximum visibility for your research: over $100 \mathrm{M}$ website views per year
}

At BMC, research is always in progress.

Learn more biomedcentral.com/submissions 